\title{
Criminologie
}

\section{Le droit de punir}

José M. Rico

Volume 19, numéro 1, 1986

Politiques et pratiques pénales. 25 ans de réflexion et d'action

URI : https://id.erudit.org/iderudit/017229ar

DOI : https://doi.org/10.7202/017229ar

Aller au sommaire du numéro

\section{Éditeur(s)}

Les Presses de l'Université de Montréal

\section{ISSN}

0316-0041 (imprimé)

1492-1367 (numérique)

Découvrir la revue

\section{Citer cet article}

Rico, J. M. (1986). Le droit de punir. Criminologie, 19(1), 113-140.

https://doi.org/10.7202/017229ar

\section{Résumé de l'article}

The main objective of this essay is to put forward some ideas in the right to punish. These ideas are put in the Canadian context and in relation to the criminal law. The criminal procedure and the criminal justice system. The first part defines the proper concepts : aims, justifications, scopes, limits and interconnections. Results cannot be properly evaluated if the basic definitions are not clear and precise. The second part presents a model for the revision and reform of criminal policies and practices. This model is based on a study of drug legislations and practices.
Ce document est protégé par la loi sur le droit d'auteur. L'utilisation des services d’Érudit (y compris la reproduction) est assujettie à sa politique d'utilisation que vous pouvez consulter en ligne.

https://apropos.erudit.org/fr/usagers/politique-dutilisation/ 
José M. Rico*

The main objective of this essay is to put forward some ideas in the right to punish. These ideas are put in the Canadian context and in relation to the criminal law. The criminal procedure and the criminal justice system. The first part defines the proper concepts : aims, justifications, scopes, limits and interconnections. Results cannot be properly evaluated if the basic definitions are not clear and precise. The second part presents a model for the revision and reform of criminal policies and practices. This model is based on a study of drug legislations and practices.

\section{INTRODUCTION}

Vers la fin des années 60 , divers comités d'enquête sur la justice pénale canadienne constataient l'absence d'une philosophie globale dans ce domaine ${ }^{1}$. La Commission de réforme du droit du Canada, créée en 1970 dans le but de «développer de nouvelles méthodes et de nouveaux concepts de droit correspondant à l'évolution des besoins de la société canadienne moderne», s'est efforcée dans plusieurs rapports et documents de travail de déterminer les objectifs du droit pénal, de la sanction et de l'ensemble du système d'administration de justice ${ }^{2}$. Cette même préoccupation inspire deux importants rapports publiés récemment par le gouvernement du Canada ${ }^{3}$.

\footnotetext{
* Professeur titulaire, École de criminologique, Université de Montréal.
}

1. Rapport du Comité canadien de la réforme pénale et correctionnelle, Justice pénale et correction: un lien à forger, Ottawa, Imprimeur de la Reine, 1969; Commission d'enquête sur l'administration de la justice en matière criminelle et pénale au Québec, la Société face au crime, 5 rapports et 9 annexes de recherche, gouvernement du Québec, Éditeur officiel du Québec, 1968-1970 (en particulier le vol. 1, Principes fondamentaux d'une nouvelle action sociale, 1968).

2. Commission de réforme du droit du Canada, Ottawa, Information Canada. Cf. notamment la Notion de blâme (document de travail 2, 1974), les Principes de la détermination de la peine et du prononcé de la sentence (document de travail 3, 1974), les Confins du droit pénal (document de travail 10, 1975) et Notre droit pénal (rapport, 1976).

3. Gouvernement du Canada, le Droit pénal dans la sociêté canadienne, Ottawa, 1982 et la Détermination de la peine, Ottawa, 1984. 
Certes, la détermination des buts et des objectifs du droit pénal, de la procédure pénale, de la sanction et de l'ensemble du système de justice constitue un souci légitime et fondamental, puisque ces principes de base serviront à l'élaboration de règles de pratique rationnelles destinées à leur application concrète dans un milieu et dans un temps donnés. À cet égard, les efforts déployés par les autorités canadiennes (aussi bien sur le plan fédéral que provincial) représentent une contribution importante dans cette voie. Cependant, il existe encore une certaine confusion ainsi qu'un manque de rigueur et d'approfondissement dans la présentation du thème.

Dans cette perspective, le but principal de cet essai est d'apporter quelques réflexions sur cet «éternel problème» du droit de punir ou jus puniendi ${ }^{4}$. Ces réflexions se réfèrent essentiellement au Canada et mettent surtout l'accent sur le droit pénal, dont les principes fondamentaux servent de base et s'appliquent aux autres domaines, objet de notre étude; elles demeurent par ailleurs j'en suis bien conscient - incomplètes et nécessitent davantage de structuration et de raffinement.

Dans une première partie, nous préciserons la portée, la signification et l'interrelation de certains termes (I). Ensuite, avec l'aide éventuelle des législations et des pratiques pénales concernant les drogues, nous esquisserons un modèle pouvant servir à la révision de l'ensemble des normes et des pratiques pénales (II).

\section{PROBLÈMES THÉORIQUES ET TERMINOLOGIQUES}

La principale confusion concernant le droit de punir tient, en premier lieu, à l'utilisation imprécise et incorrecte de certains termes (droit pénal, procédure pénale, peine, système de justice pénale), souvent considérés comme des synonymes alors qu'ils

4. I'ai déjà abordé ce thème dans les publications suivantes: «Les législations hispano-américaines de dangerosité sociale: évolution et signification», dans Ch. Debuyst (édit.), Dangerosité et justice pénale, Genève, Masson, 1981, p. 231-258 (reproduit aussi dans la Revista Mexicana de Ciencias Penales, année III, n. 3, juillet 1979-juin 1980, p. 291-318); "Alternativas al castigo», Annales internationales de criminologie, 1983, vol. 21, n. 2, p. 313-324; «Las legislaciones sobre drogas: origen, evolucion, significado y replanteamientos», rapport présenté au XXXV Cours international de criminologie sur le thème «Alcool, drogues et criminalité», Quito, Équateur, 13-18 août 1984, 58 p. (la version française sera publiée dans un prochain numéro de la revue Psychotropes). 
réfèrent à des niveaux de réalité différents ${ }^{5}$ et, en second lieu corollaire vraisemblable de cette première lacune -, à l'assignation d'objectifs inadéquats à plusieurs de ces termes ${ }^{6}$. Cette confusion rend aléatoire tout effort visant à une correcte détermination des objectifs des institutions concernées et, par conséquent, compromet sérieusement les résultats attendus par leur mise en pratique.

Il est donc essentiel de définir chacun des termes utilisés et d'indiquer leur justification et leurs objectifs, ainsi que leur portée et leurs limites.

\section{A. LE DROIT PÉNAL}

\section{Définition}

Le droit pénal peut être envisagé dans un double sens : comme un ensemble de mesures relatives aux délits et aux peines (droit pénal objectif ou jus poenale) et comme la faculté ou le pouvoir de l'État d'interdire certains agissements de nature à créer un trouble grave dans la société et d'imposer des sanctions à leurs auteurs (droit pénal subjectif ou jus puniendi) ${ }^{7}$.

Le droit pénal comprend deux grandes parties : le droit pénal général et le droit pénal spécial. Le droit pénal général contient les principes généraux concernant les délits, leurs auteurs et les peines. En ce qui a trait aux deux premiers points, il définit le délit, précise ses éléments fondamentaux (légal, matériel et moral) ainsi que ses modes d'apparition, et détermine le degré de responsabilité pénale des infracteurs. Le droit pénal général comprend

5. Ceci peut être attribué à l'absence relative de développements théoriques concernant la philosophie pénale caractéristique de la famille juridique anglo-saxone;

6. Ainsi, le Droit pénal dans la société canadienne (op. cit., note 3 , p. 43-48) utilise indistinctement les expressions «droit pénal» et «justice pénale» dass le chapitre consacré à la nature du droit pénal, alors que la Commission de réforme du droit du Canada énumère, parmi les objectifs de celui-ci, la liberté et la justice, qui correspondent davantage à la procédure pénale (Notre droit pénal, op. cit., note 2, p. 8-9), ou bien le châtiment, la dissuasion et la réhabilitation, qui constituent des objectifs possibles de la peine (les Confins du droit pénal, ibid., p. 39-40; Notre droit pénal, ibid., p. 3).

7. En ce sens José M. Rodriguez Devesa, Derecho penal espanol. Parte general, 8 éd., Madrid, Artes Graficas Carasa, 1981, p. 5-43. 
également des règles relatives à la fixation de la peine et autres mesures de nature pénale, notamment l'énumération de celles-ci et les modalités de leur application. Le droit pénal spécial, qui historiquement a précédé le droit pénal général et représente la principale raison d'être du droit de punir, se limite à décrire les diverses infractions et à déterminer les peines à infliger à chacune d'elles'.

\section{Justification}

Pendant de longs sjècles, le droit de punir - et en particulier le droit pénal spécial - a été justifié soit par une référence à la divinité (toutes les choses ont leur origine en Dieu), soit par le recours au droit naturel (suivant lequel il existe des impératifs moraux qui «obligent» le Prince à ne pas laisser impunis certains faits particulièrement odieux). Cependant, l'existence hypothétique de valeurs permanentes et immuables attribuables soit au caractère divin de la création, soit à la transcendence de l'homme par rapport à la nature n'est qu'un problème philosophique sans traduction possible en termes objectifs. Par ailleurs, les normes qui composent le droit naturel sont en réalité le reflet des valeurs de ceux qui les «découvrent» et les élaborent. De là le caractère idéologique de ce droit; c'est le détenteur du pouvoir qui décide, en dernière instance, de son contenu'.

Sans prétendre nier l'existence de certaines valeurs fondamentales propres à notre civilisation, la seule justification valable du droit de punir réside actuellement dans la loi pénale, émanation de la souveraineté (jus imperium) de l'État. En effet, on ne peut pas concevoir de nos jours que l'État, dont l'une des fonctions essentielles et traditionnelles est la tutelle de l'ordre juridique, grâce auquel sont assurées la coexistence pacifique du groupe humain et l'harmonie des rapports entre les membres de la communauté, ne dispose pas des moyens nécessaires pour garantir le respect des lois et des biens juridiques dont la sauvegarde est sa raison d'exister. Sans la reconnaissance de ce droit - et tout en acceptant dès le départ que tous les conflits sociaux ne peuvent pas être réglés par l'intervention répressive de l'État -, l'ordre

8. De façon concrète, le droit pénal comprend le code pénal et l'ensemble des lois de nature punitive.

9. Luis de Ia Barreda Solorzano, «lus puniendi y ius poenale», Capitulo Criminologico 9-10, 1981-1982, p. 69-94. 
juridico-pénal d'un pays manquerait de légitimitét ${ }^{10}$. Ce sont, en somme, les nécessités sociales qui justifient, sous certaines conditions que nous examinerons plus loin, l'action législative des détenteurs du pouvoir". La loi pénale étant toutefois un produit spirituel de l'homme, c'est-à-dire faillible et perfectible, toute approche exclusivement dogmatique est à exclure dans ce domaine; par conséquent, la voie de la critique du droit pénal doit toujours être ouverte ${ }^{12}$.

\section{Objectifs}

Ainsi, le principal objectif du droit pénal est d'assurer la coexistence pacifique des citoyens en protégeant de façon rigoureuse certains biens juridiques ${ }^{13}$, ce qui implique une aspiration éthico-sociale ${ }^{14}$. Néanmoins, le droit pénal ne prétend pas régir toute l'éthique sociale; il ne le pourrait d'ailleurs pas.

Cette protection de certains biens juridiques (ou valeurs) fondamentaux et socialement acceptés ${ }^{15}$ s'effectue notamment à l'aide d'un ensemble d'interdictions et de sanctions destinées à réagir de façon juste et appropriée devant diverses conduites

10. «Infliger une peine n'est pas un événement métaphysique, mais une amère nécessité dans une communauté imparfaite telle la communauté humaine» (Projet alternatif de Code pénal de la RFA, 1965). Contre cette interprétation, cf. les thèses des utopistes (Th. More), des anarchistes, des marxistes, des abolitionnistes et de certains auteurs (Tolstoï, A. France, Cervantes, etc.).

11. «Le droit pénal... constitue une réponse nécessaire à des comportements répréhensibles» (Notre droit pénal, op. cit., note 2, p. 16).

12. Selon la Commission d'enquête sur l'administration de la justice au Québec (op. cit., note 1), l'un des principes fondamentaux pour une nouvelle action sociale dans le domaine de la justice pénale devrait être l'existence d'une loi moderne, représentant vraiment les volontés profondes de la population et sachant tenir compte rapidement de l'évolution de la société.

13. L'objectif de sécurité du rapport le Droit pénal dans la société canadienne, op. cit., note 3, p. 46.

14. E. Raul Zaffaroni, Tratado de derecho penal. Parte general, Buenos Aires, Ediar, 1980, p. 50.

15. Cet objectif correspond à l'idéal d'humanité retenu par la Commission de réforme du droit du Canada (Notre droit pénal, op. cit., note 2, p. 7-8). Cet idéal d'humanité se manifesterait par la condamnation de certains comportements (meurtre, viol, vol avec violence et autres délits du même genre) qui s'opposent au respect mutuel que notre société humaniste impose comme condition essentielle à la vie en société. 
susceptibles de causer un grave préjudice aux personnes ou à la collectivité ${ }^{16}$.

Par ailleurs, en réaffirmant les valeurs sociales essentielles d'une société, en imposant leur respect et en condamnant leur violation, le droit pénal joue un important rôle moral et éducatif ${ }^{17}$.

Mais, quels sont - ou devraient être - ces biens juridiques (ou valeurs) que l'État doit sauvegarder d'une façon si rigoureuse? Leur détermination pose le problème de la portée et des limites du droit pénal.

\section{Portée et limites}

Il convient tout d'abord de rappeller que, dans nos sociétés démocratiques contemporaines, seul l'État détient le jus puniendi. Par conséquent, la fixation des valeurs à protéger pénalement est une décision politique qui dépend des conceptions éthico-sociales dont se prévalent les personnes et/ou les groupes au pouvoir à un moment donné de l'évolution historique d'un pays.

Cependant, dans un État de droit, cette intervention politique ne doit pas être de nature partisane et conjoncturelle mais se baser sur les grands principes inscrits dans la constitution du pays, qui jouissent d'une certaine stabilité et ont été généralement acceptés par tous les groupes politiques représentant la communauté. Ces principes constitutionnels font eux aussi partie de l'ordre juridique de chaque pays, dont ils occupent le rang le plus élevé. Ils sont, par ailleurs, des principes à la fois politiques et éthico-sociaux. Politiques, dans la mesure où ils représentent un certain consensus entre les forces politiques, qui, à leur tour, s'engagent à les respecter. Éthico-sociaux, étant donné qu'avant d'atteindre le haut rang qu'ils occupent désormais, ils constituaient une espèce de code de base de ce que dans la vie sociale était considéré bon ou mauvais ${ }^{18}$.

16. C'est ainsi que le rapport du gouvernement du Canada le Droit pénal dans la société canadienne (note 3, p. 5, 61 et 65) définit l'objet du droit pénal.

17. En ce sens Notre droit pénal, note 2, p. 6.

18. Luis Rodriguez Ramos, «Criterios politicos y técnicos para la creacion y abrogacion de las normas penales», Revista Mexicana de lusticia, n. 1, vol. 1, janvier-mars 1983 , p. $37-66$ (surtout p. 52). Quelques aspects de ce qui va suivre s'inspirent de cet article. 
Au Canada, la Charte des droits et des libertés de 1981 reconnait explicitement les principes suivants reliés au thème que nous abordons : la primauté du droit (préambule); les droits à la vie, à la liberté et à la sécurité de la personne (art. 7); la présomption d'innocence (art. 11, d); la légalité (art. 11, g); la protection contre tout traitement ou peine cruel et inusité (art. 12); et un certain nombre de garanties juridiques relatives au procès (art. 8 à 14).

Ces principes explicites constituent, par ailleurs, la base d'autres principes implicites de grande importance. Parmi ces derniers, il convient de souligner le principe de l'intervention minime, celui de l'intervention non stigmatisante et celui de la sécurité juridique.

En ce qui concerne le principe de l'intervention minime, sa justification repose sur la reconnaissance des droits fondamentaux déjà énumérés (dignité de la personne, liberté, sécurité). Ceux-ci limitent l'action du législateur dans la mesure où les prohibitions et les sanctions pénales supposent inévitablement des restrictions de la liberté et d'autres droits fondamentaux. Le droit pénal constitue donc l'ultima ratio du législateur; ceci signifie que l'on doit exclure de son champ d'action les cas impliquant des valeurs pour la protection desquelles il suffit de recourir à d'autres institutions ou mécanismes sociaux tels la famille, l'école, la collectivité. Le droit pénal ne peut être qu'une solution partielle et ne peut jouer qu'un rôle limité dans le règlement des conflits sociaux ${ }^{19}$. Son utilisation dans ce dernier but représente non seule-

19. Cf. Notre droit pénal, note 2, p. 15-19, 27-28 et 33. Dans le même sens. les Confins du droit pénal, note 2, p. 57 («le droit pénal ne constitue qu'une arme dans tout l'arsenal des lois»; «il faut toujours se rappeler la rançon qui se rattache au droit pénal : souffrances, pertes de liberté et coût d'ordre financier»), ainsi que le Droit pénal dans la sociésé canadienne, note 3 ( il faut, avant de recourir à ce moyen ultime que représente le droit pénal, se demander sérieusement s'il est approprié, nécessaire et préférable aux autres méthodes à caractères moins coercitif et portant moins gravement atteinte aux droits individuels, pour régler divers problèmes sociaux», p. 48-49; «le droit pénal devrait être conçu comme le moyen ultime dont dispose la société parmi toute une série d'usages et d'institutions empreints d'un formalisme plus ou moins grand», p. 48; "on ne doit recourir au droit pénal que lorsque d'autres moyens d'intervention sociale sont inadéquats ou inappropriés à l'égard d'un certain mode de comportement et de façon à n'empiéter qu'au minimum sur les droits et libertés des personnes», p. 5, 61 et 67; «il est injuste d'aller au-delà de l'intervention minime nécessaire pour réagir de façon adéquate et appropriée à un comportement criminel, 
ment le plus négatif des recours, si l'on tient compte des privations ou des restrictions de droits inhérents à la peine, mais également un recours pessimiste, car le droit pénal n'intervient que lorsque toutes les mesures de prévention n'ayant pas de caractère répressif ont été déployées sans succès ${ }^{20}$. 11 faut, par conséquent, l'appliquer avec modération; il faut aussi simplifier les normes pénales existantes.

Le principe de l'intervention non stigmatisante comporte deux aspects essentiels : le recours à d'autres voies que la voie répressive (droit civil, droit administratif, arbritrage, etc.), et l'utilisation moins discriminatoire du droit pénal (traditionnellement appliqué aux secteurs les plus défavorisés de la population ${ }^{21}$.

Enfin, le principe de la sécurité juridique consiste à décrire avec précision et avant leur application éventuelle la portée des normes pénales (nullum crimen, nulla poena sine lege), à refuser l'irrétroactivité de la loi pénale et à garantir les libertés individuelles lors du procès.

C'est par rapport à ce cadre général que se pose le problème de la détermination précise des biens juridiques à protéger pénalement. Si, pour les raisons que nous venons d'exposer, les normes pénales ne devraient être envisagées que dans la mesure et dans les cas où elles sont nécessaires pour assurer les conditions qui rendent possible la coexistence sociale pacifique, seules devraient faire l'objet d'incrimination les conduites qui les mettent sérieuse-

que ce soit pour des motifs d'utilité ou de rétribution», p. 4 et $57-58$ ). Déjà en 1969, le Comité canadien de la réforme pénale et correctionnelle (op. cit., note 1, p. 11-19) stipulait ce qui suit : «les objets fondamentaux du droit pénal doivent se réaliser sans empiéter plus qu'il n'est nécessaire sur la liberté des individus»: «aucune conduite ne doit être décrite comme criminelle, sauf... s'il est impossible de la redresser par d'autres moyens sociaux ou légaux».

20. E. Ramirez Hernandez, «Fuentes reales de las normas penales», Revista Mexicana de Justicia, n. 1, vol. 1, janvier-mars 1983, p. 23-35 (en particulier, p. 27); pour cet auteur, le ius poenale signifie «un effort désespéré pour éviter le chaos».

21. Cette solution est préconisée par des auteurs appartenant à la «criminologie critique» (Lolita Aniyar de Castro, Alessandro Baratta, Louk Hulsman, etc.). Néanmoins, le droit pénal a toujours été et demeure essentiellement une institution à caractère punitif» (Cf. le Droit pénal dans la société canadienne, note 3 , p. 46); il semble également illusoire et peu conforme avec la réalité historique de songer à l'abolition complète du système pénal. 
ment en danger. Après avoir décrit avec précision ces conduites, il faudrait établir entre elles une certaine stratification ou hiérarchie, en tenant compte des valeurs qu'elles impliquent.

En ce qui concerne les comportements à interdire pénalement, nous avons déjà indiqué qu'ils doivent être de telle nature qu'ils soient susceptibles de causer un grave préjudice aux personnes ou à la collectivité. C'est dans ce sens que la Commission de réforme du droit du Canada utilise les expressions "crimes véritables» ou «infractions qui vont à l'encontre des valeurs fondamentales»"2. La détermination exacte de ces valeurs fondamentales ne semble pas poser des problèmes lorsqu'il s'agit de certaines valeurs universellement reconnues et faisant partie essentielle de notre patrimoine culturel (la vie, l'intégrité physique, la vérité, l'ordre juridicoconstitutionnel) ; la violation de ces valeurs fondamentales constitue des délits tels le meurtre, les blessures, le viol, le vol avec violence sur les personnes, la fraude, la trahison, la rebellion, etc. ${ }^{23}$. En plus de ces biens fondamentaux et universels, certaines sociétés en font primer d'autres. Ainsi, les sociétés occidentales accordent une importance considérable à la liberté individuelle, à la dignité humaine, à la santé, à la justice, à la tolérance, à l'égalité et à la propriété privée; des délits tels la séquestration arbitraire, la propagande haineuse et raciale, le commerce des drogues, l'entrave à la justice et le vol constituent des violations de ces importantes valeurs ${ }^{24}$. Cette distinction représente un critère valable pour l'établissement d'une hiérarchie parmi les biens juridiques mentionnés. Par ailleurs, les auteurs des infractions graves ne devraient être condamnés que s'ils ont agi intentionnellement ou avec insouciance ${ }^{25}$.

La reconnaissance de ces biens juridiques traditionnels n'exclut pas, bien entendu, la possibilité d'incriminer des compor-

22. Cf. Notre droit pénal, note 2, p. 19, 28 et 34 . Dans le même sens, le rapport du Comité canadien cité, note 1, p. 11-19 («aucune conduite ne doit être décrite comme criminelle, sauf si elle constitue une grave menace pour la société»), ainsi que celui du gouvernement canadien, note 3, p. 53 («le droit pénal doit être réservé au comportement qui est hautement nuisible», c'est-à-dire qui a causé ou peut avoir causé un préjudice à la société ou à l'intégrité physiqưe des personnes ou de leurs biens).

23. Notre droit pénal, p. 21. Également, le Droit pénal dans la société canadienne, note 3, p. 49.

24. Notre droit pénal, p. 21.

25. Ibid., p. 23 et 29, et le Droit pénal dans la société canadienne, p. 55. 
tements (individuels ou collectifs) qui, à un moment donné, causent - ou peuvent causer - un tort grave aux personnes ou à la communauté. C'est ainsi que, de nos jours et dans des sociétés comme la notre, il est question de sanctionner pénalement des conduites telles la pollution, l'épuisement des ressources, l'exploitation, le racisme, certaines activités des multinationales, etc.

Par contre, et en application des principes déjà exposés, toutes les infractions qualifiées de "réglementaires» devraient être exclues du champ pénal ${ }^{26}$. On devrait également décriminaliser partout où la prohibition n'est pas nécessaire, opportune et efficace, et substituer à la poursuite pénale des solutions de rechange plus constructives, principalement axées sur la collectivitén.

Un dernier pas reste à faire. Une fois que la présence d'un bien juridique digne de protection a été déterminée, il faut ensuite établir si toutes les conduites qui peuvent l'attaquer doivent être prohibées, ou bien s'il convient d'en faire une sélection appropriée. Par exemple, le fait de considérer la santé (individuelle ou collective) comme un objet digne de protection en matière de drogues suppose que l'on doive s'interroger sur la nécessité d'avancer ou de reculer les barrières d'une telle protection, en incriminant les plus éloignées ou hypothétiques (la culture de la substance, voire la cession d'un terrain pour sa plantation), ou uniquement celles qui paraissent avoir une étroite connexion avec le dommage prévu (le commerce, la possession, l'usage).

\section{B. LA PEINE}

\section{Définition}

La peine peut être définie comme la privation ou la restriction d'un bien juridique (vie, liberté, propriété, etc.) établie par la loi et appliquée par l'organisme judiciaire compétent à toute personne

\section{Notre droit pénal, p. 22.}

27. Ibid., p. 31. À la lumière de ces critères, la Commission de réforme du droit du Canada recommande une étude spéciale de trois catégories d'infractions, en vue d'une possible décriminalisation : a) les actes que la majorité des gens ne considèrent pas suffisamment graves pour qu'on les incrimine pénalement (paris, magie, etc.); b) ceux dont le caractère répréhensible et la gravité font l'objet de controverses (avortement, obscénité, prostitution, drogues, inceste, etc.); et c) les infractions relatives à la proprieté (ibid., p. 35-36). 
déclarée coupable de la commission d'un délit à la suite d'un procès juste et équitable ${ }^{28}$.

La problématique de la peine fait partie de celle concernant le droit pénal, qui est un droit fondamentalement punitif. Elle présente toutefois de nombreuses particularités, notamment en ce qui a trait à sa justification, ses objectifs, sa portée et ses limites.

\section{Justification}

Sauf quelques exceptions, le droit de l'État à imposer des sanctions pénales est un droit reconnu universellement ${ }^{29}$. Par contre, sa justification a toujours fait l'objet de sérieuses controverses.

Les théories absolues cherchent le fondement et le but de la peine, non pas dans un objectif transcendant mais dans la nature intime de celle-ci. On punit quia peccatur est, parce que l'on a commis un délit. La peine est juste en elle-même, indépendamment de son utilité éventuelle. La sanction est purement et simplement la conséquence juridique de l'infraction. Selon ces théories, le but de la peine est la rétribution et l'expiation de la faute commise.

Par contre, les théories relatives assignent à la peine un but politique et utilitaire. On punit ut ne peccetur, c'est-à-dire afin d'éviter la commission de nouveaux délits. La peine est imposée en raison de son efficacité, compte tenu de ses résultats probables et de ses effets.

Les théories relatives se divisent en plusieurs groupes. Le groupe le plus important est celui qui attribue à la peine l'objectif de prévenir la commission de nouveaux délits (théories préventives); d'autres groupes lui assignent les fonctions de réparer les conséquences nuisibles de l'acte, de réconcilier l'auteur, la victime et la communauté, de neutraliser les éléments les plus dangereux de la société et de réhabiliter le délinquant. À leur tour, les théories

28. José M. Rico, Las sanciones penales y la politica criminologica contemporanea, ze éd., Mexico, Siglo XXI, 1984 p. 9.

29. Selon E. Novoa Monreal («Se justifica el derecho a castigar?», Annales internationales de criminologie, 1983, vol. 21, n. 2, p. 171-185), aucun État n'a jamais renoncé jusqu'à présent à exercer le droit de punir et on ne voit pas de possibilités réelles qu'il le fasse dans le futur; par ailleurs. s'il le faisait, des réactions subjectives de la population devant certains dêlits particulièrement graves seraient à craindre. 
préventives se sous-divisent selon qu'elles poursuivent un objectif de prévention générale ou de prévention spéciale. Celles qui défendent la prévention générale utilisent la peine par référence à la collectivité : la sanction pénale, en raison des conséquences qu'elle entraîne, accomplit un important effet intimidant sur l'ensemble de la population, évitant de la sorte la commission de nouveaux délits. Les théories qui prétendent atteindre un but de prévention spéciale utilisent la sanction uniquement par rapport à l'infracteur et considèrent que son exécution est le seul moyen d'éviter la récidive.

Finalement, les théories mixtes essaient de concilier les points de vue de la justice absolue et d'une fin utilitaire.

Les limites de cet essai nous empêchent de procéder à l'analyse critique et approfondie de chacune de ces théories ${ }^{30}$. Il convient cependant de souligner la confusion qui règne depuis longtemps dans ce domaine. Cette confusion se manifeste tant sur le plan de la justification de la peine que sur celui de la détermination de ses objectifs.

\section{Objectifs}

Ceux-ci viennent d'être énumérés. Il s'agit de la rétribution ${ }^{31}$, de l'expiation, de la neutralisation, de l'intimidation (générale et spéciale), de la réparation du dommage, de la conciliation et de la réhabilitation ${ }^{32}$. Il convient d'y ajouter la fonction morale et éducative de la peine, si importante pour la restauration de la tranquillité publique dans les cas de délits particulièrement graves.

Or, la majorité des peines poursuivent plusieurs objectifs, souvent contradictoires voire irréconciliables, qui dépendent en grande partie du rôle spécifique assigné à l'organisme ou à la personne chargée de son application. C'est ainsi que le législateur,

30. Pour une étude plus détaillée de ces théories ainsi que des objectifs des diverses mesures pénales, cf. José $M$. Rico, op. cis., note 28, p. 9-47.

31. L'imposition d'un juste châtiment ou d'une peine méritée, selon la Commission de réforme du droit du Canada (la Détermination de la peine, note 3, p. 39).

32. Cf. les Principes de la détermination de la peine et du prononcé de la sentence, note 2, p. 3-4, ainsi que la Détermination de la peine, note 3. p. 6.9 et le Droit pénal dans la société canadienne (note 3, p. 6, 62 et 70-71), qui fait référence à la conciliation, à la compensation et à la réhabilitation (cependant, ces objectifs sont attribués au droit pénal et au système de justice pénale, et non pas à la peine). 
qui agit généralement à un niveau abstrait, vise en principe la prévention générale, tandis que le juge intervenant surtout dans des cas concrets, poursuit principalement la prévention spéciale à travers l'individualisation de la sentence, et que l'administration pénitentiaire prétend essentiellement obtenir la réinsertion sociale de la personne condamnée.

Cette confusion peut être attribuée à l'absence de critères et d'indications explicites qui puissent servir à l'identification précise des objectifs généraux de la sanction pénale, ainsi qu'à celle des objectifs spécifiques de chacune des peines ${ }^{33}$. Par ailleurs, elle peut expliquer l'inefficacité de la plupart de ces objectifs, en particulier ceux de la réhabilitation, de la dissuasion et de la neutralisation par l'emprisonnement ${ }^{34}$.

Néanmoins, il ne convient pas d'oublier que les sanctions pénales sont avant tout punitives et perçues comme telles à la fois par la population et par ceux qui les subissent $t^{35}$. Ceci ne signifie pas qu'elles ne puissent pas se justifier également par des motifs utilitaires (c'est-à-dire en faisant appel aux divers objectifs de cette nature), ni qu'elles doivent être dures, cruelles ou dégradante ${ }^{36}$ Voici déjà quelques limites de la peine.

\section{Portée et limites}

Le recours à la sanction pénale doit toujours dépendre de considérations politico-criminelles de nécessité, d'opportunité, de justice, d'efficacité et de coûts sociaux.

La peine doit d'abord être nécessaire et opportune. Le principe de l'intervention minime, valable quant au droit pénal, doit également être appliqué dans ce domaine. Dans cette perspective, en imposant une sentence, le juge doit choisir la mesure la moins restrictive qui soit suffisante et adéquate aux circonstances ${ }^{37}$, faire preuve de modération (en particulier dans l'imposition d'une

33. Cf. les Principes de la détermination de la peine et du prononcé de la sentence, note 2, p. 27 , ainsi que la Détermination de la peine, note 3 , p. 37.

34. José M. Rico, note 28, p. 9-47. Dans le même sens, la Détermination de la peine, note 3, p. 6 .

35. Le droit pénal dans la société canadienne, note 3, p. 3.

36. Ibid. Voir également les Principes de la détermination de la peine et du prononcé de la sentence, note $2, \mathrm{p} .3$.

37. Le Droit pénal dans la société canadienne, note 2, p. 6, 62 et 72, et La détermination de la peine, note 2, p. 42. 
sanction privative de liberté), utiliser davantage les mesures non carcérales (surtout celles axées sur la collectivité) et accorder une plus grande attention aux préoccupations des victimes ainsi qu'à celles de la communautét ${ }^{38}$.

La peine doit ensuite être juste et équitable. Il ne suffit pas que "justice soit faite»; l'infracteur, la victime et la communauté doivent en être profondément convaincus. À cet égard, on doit éviter les incohérences (ou disparités judiciaires) dans le prononcé de la sentence ${ }^{39}$, grâce à l'élaboration et à l'application d'un ensemble de lignes directrices qui, tout en laissant une certaine marge de discrétion au juge, les éliminent ou les réduisent considérablement ${ }^{40}$; en application de ce principe, les infractions semblables devraient être traitées de la même façon ${ }^{41}$. On doit aussi veiller à ce que les sanctions à infliger soient proportionnelles à la gravité objective cie l'infraction, aux risques de préjudice (ou au préjudice effectivement subi) et au degré de culpabilité et de responsabilité du contrevenan $t^{42}$.

La sanction doit également être efficace, c'est-à-dire atteindre les objectifs pour lesquels elle a été prévue. Or, nous avons déjà indiqué que les résultats obtenus à cet effet, pour les raisons mentionnées, sont loin d'être satisfaisants. On doit donc améliorer avec précision les buts assignés à chacune des mesures pénales, améliorer la structure actuelle des peines (grâce à l'acceptation de nouvelles mesures, ainsi qu'au regroupement, à la stratification hiérarchique et à la rationalisation des dispositions existantes), réviser les maximums et les minimums prévus pour chaque infraction ainsi que les sanctions minimales obligatoires (afin surtout de rendre les mesures pénales plus réalistes), apporter davantage de clarté aux normes du code pénal relatives aux objectifs de la peine

38. La Détermination de la peine, p. 5 et 35-36.

39. À ne pas confondre avec une disparité imposée par le principe de l'individualisation de la peine; $c f$ à cet égard l'ouvrage cité dans la note précédente, p. 15-16.

40. Ibid., p. $22-24$ et 35 .

41. Les principes de la détermination de la peine et du prononcé de la sentence, note 2, p. 3; le Droit pénal dans la société canadienne, note 3, p. 6, 62 et 72; la Détermination de la peine, note 3, p. 42.

42. Ibid., p. $3 ; 6,62$ et $70 ; 41-42$. 
et à ses modalités d'exécution et prendre en considération l'effet total de la peine (lorsque la cour impose plusieurs sanctions ou des sanctions consécutives à l'infracteur) ${ }^{43}$.

Enfin, on doit tenir compte des coûts économiques, financiers, sociaux et moraux que la peine entraine en général et que chacune des sanctions suppose. Dans ce domaine, la règle devrait être la minimisation des coûts, que l'on pourrait obtenir grâce à l'apport de la communauté, c'est-à-dire en faisant siéger des citoyens avec le juge afin de participer à la détermination de la sentencet.

\section{LA PROCÉDURE PÉNALE}

\section{Définition}

La procédure pénale fait également partie du droit pénal considéré au sens large. Celui-ci est un droit essentiellement judiciaire; il ne peut en principe recevoir d'application que par un procès intenté par la société, dont l'ordre a été troublé, contre l'auteur de ce trouble. L'organisation des différentes juridictions pénales, leur compétence et leurs règles de fonctionnement, ainsi que le déroulement de ce procès, depuis les premières investigations de la police jusqu'à la décision finale et les voies de recours qui peuvent être prises contre elles sont régis par des règles dont l'ensemble constitue ce que l'on appelle la procédure pénale. Celle-ci a donc pour objet la réglementation du procès pénal ${ }^{45}$.

\section{Justification}

En ce qui concerne le phénomène de la criminalité, la collectivité ne peut pas manquer de réagir contre ceux qui méconnaissent les plus fondamentales des règles posées par l'autorité compétente pour l'harmonisation des rapports sociaux. Cependant, la réaction des pouvoirs publics ne peut pas être la réaction brutale et aveugle de la victime ou de ses proches; elle doit être organisée et adaptée à certaines fins. Le fait même que plusieurs personnes aient contrevenu aux règles reconnues, introduit déjà dans leurs con-

43. Cf. la Détermination de la peine, p. 24-37 et 42.

44. En ce sens les Principes de la détermination de la peine et du prononcé de la sentence, note $2, \mathrm{p} .1$.

45. G. Stefani et G. Levasseur, Droit pénal général et procédure pénale, 2 t., 2e éd., Paris, Dalloz, 1966, t. 1, p. 37.39 et t. 2, p. 1.2. 
duites un élément juridique. C'est encore sur le terrain juridique que la réaction contre l'activité criminelle doit se développer, à moins de conduire à l'anarchie ou à la tyrannie; elle le fera donc selon certaines règles préétablies. Elle le fera également dans le cadre de certaines structures, qui dépendent de l'organisation politique de chaque pays, ainsi que de la politique criminelle que celui-ci entend suivre.

\section{Objectifs}

La procédure pénale poursuit deux objectifs essentiels. D'un côté, la défense de la société, atteinte par l'infraction, moyennant l'établissement de rèlges qui rendent possible la découverte rapide, le jugement et la condamnation de ceux qui ont enfreint la loi pénale. De l'autre, elle doit garantir les libertés des individus et les droits de la défense, sans le respect desquels il ne saurait y avoir une vraie justice. L'équilibre entre ces deux buts fondamentaux constitue l'idéal vers lequel tendent les systèmes de justice des pays les plus progressistes.

Le Canada s'inscrit dans cette orientation philosophique. "Le but premier du processus pénal est de déterminer la culpabilité ou l'innocence des accusés» ${ }^{46}$. Cependant, cette "recherche de la vérité» par le procès pénal n'a pas une valeur absolue. Le respect de la dignité humaine et de la vie privée et la protection contre le risque de condamner des innocents constituent à la fois ses plus importants objectifs et limites ${ }^{47}$.

\section{Portée et limites}

De façon plus spécifique, le procès pénal doit obéir aux principes suivants : légalité, égalité de tous devant la loi pénale, équité, présomption d'innoncence, doute raisonnable et respect des droits de la défense (en particulier grâce à la protection contre l'arrestation et la détention arbitraires ainsi que contre les peines cruelles et inusitées, et au droit à une audience devant un arbitre indépendant et impartial) ${ }^{48}$.

46. Commission de réforme du droit du Canada, Procédure pénale. La communication de la preuve, document de travail 4, Ottawa, Information Canada, 1974, p. 5.

47. Ibid., p. 5-6.

48. Cf. Notre droit pénal, note 2, p. 7.9 et le Droit pénal dans la société canadienne, note 3, p. 5.6, 61-62 et 69 . 


\section{LE SYSTÈME DE JUSTICE PÉNALE}

L'expression "système de justice pénale» recouvre toute une organisation composée d'éléments multiples et variés (textes, institutions, personnes, idées, activités, etc.) situés les uns par rapport aux autres de façon stable mais en constante interaction. En ce sens, elle comprend: a) le droit pénal en tant qu'un ensemble de textes, de doctrines et d'idées; b) un certain nombre d'organismes publics et privés (Parlement, ministères de la Justice et de l'Intérieur, police, Défense, ministère public, tribunaux, prisons, services de réhabilitation, etc.) dont l'existence et le fonctionnement sont reliés au problème criminel et à la réaction sociale qu'il suscite ${ }^{49}$.

En raison de l'amplitude de son champ d'action, la justification, les objectifs, la portée et les limites du système de justice pénale correspondent à ceux des diverses institutions qui le composent. La principale particularité dans ce domaine réside dans leur harmonisation et la prise en considération du caractère de totalité du système pénal ${ }^{50}$. En plus, dans la mesure où nous nous référons à un système démocratique, il est capital d'instaurer des mécanismes appropriés de surveillance de son fonctionnement et de participation de la collectivitét .

\section{MODÈLE POUR L'INTERPRÉTATION ET LA RÉVISION DES NORMES ET DES PRATIQUES PÉNALES}

L'analyse que nous venons de faire nous a permis de définir et de situer correctement les diverses composantes du droit de punir. Elle nous permet également de dégager les principes de base pouvant servir à l'élaboration d'un modèle pour l'interprétation et la révision des normes et des pratiques pénales.

49. Jacqueline Bernat de Celis, «Les grandes options de la politique criminelle : la perspective de Louk Hulsman», Archives de politique criminelle, 1982, n. 5, p. 15; Louk Hulsman et Jacqueline Bernat de Celis, Peines perdues : le système pénal en question, Paris, Le Centurion, 1982, p. 60-62.

50. Dans ce sens le rapport du Comité canadien de la réforme pénale et correctionnelle, op. cit., note 1 («le maintien de l'ordre, le processus judiciaire et les mesures de redressement devraient être étroitement rélié»). 73.75 .

51. Cf. le Droit pénal dans la société canadienne, note 3, p. 6, 62 et 
Dans ce but, nous allons d'abord présenter ce modèle; puisque ses principaux éléments ont déjà été exposés, nous le ferons de façon schématique. Ensuite, nous le soumettrons à l'épreuve dans un terrain concret : les législations et les pratiques pénales concernant les drogues ${ }^{52}$.

\section{A. $L E M O D \grave{E} L E$}

\section{Le droit pénal}

- la seule justification valable du droit pénal réside dans la loi, émanation de la souveraineté de l'État;

- ce sont les nécessités sociales qui légitiment l'action législative des détenteurs du pouvoir;

- étant donné que les nécessités sociales peuvent changer et que la loi pénale est un produit spirituel de l'homme (donc faillible et perfectible), toutes deux doivent faire l'objet de critiques, d'évaluations et de révisions périodiques, surtout dans le but d'adapter la loi aux besoins réels de la communauté;

- l'objectif fondamental du droit pénal est d'assurer la coexistence pacifique des membres de la collectivité en protégeant de façon rigoureuse certains biens juridiques essentiels et socialement acceptés; cette protection se réalise à travers l'interdiction pénale de diverses conduites ou omissions susceptibles de causer un grave préjudice aux personnes ou à la collectivité; éducatif;

- le droit pénal joue aussi un important rôle moral et

- le droit pénal général doit définir le délit, préciser ses éléments fondamentaux (légal, matériel, moral) ainsi que ses modes d'apparition, déterminer le degré de responsabilité pénale des infracteurs, établir la liste des sanctions et autres mesures pénales et fixer ses modalités d'application;

- le droit pénal spécial doit décrire les diverses infractions et déterminer les peines à infliger à chacune d'elles;

- le droit pénal doit se baser sur les grands principes inscrits dans la constitution du pays;

52. Pour ce faire, nous aurons recours à notre essai sur ce sujet indiqué à la note 4 . 
- le droit pénal représente l'ultima ratio du législateur; il ne doit donc être utilisé que si d'autres mécanismes sociaux ont été déployés sans succès pour le règlement de certains conflits graves;

- le droit pénal ne doit pas être conçu et appliqué de façon stigmatisante et discriminatoire;

- les conduites à interdire pénalement doivent être décrites avec précision;

- pour qu'une conduite soit interdite pénalement, elle doit représenter un grave préjudice aux personnes ou à la collectivité, ainsi que la violation de certaines valeurs universellement reconnue et faisant partie essentielle du patrimoine culturel du pays; dans des sociétés comme la nôtre, la vie, l'intégrité physique, la vérité, l'ordre juridico-constitutionnel, la liberté individuelle, la dignité humaine, la santé, la justice, la tolérance, l'égalité et la propriété privée font partie de ces valeurs fondamentales;

- l'incrimination pénale ne doit porter que sur les actions ayant une étroite connexion avec le dommage prévu, évitant celles plus hypothétiques ou éloignées.

\section{La peine}

- la justification et les objectifs possibles de la peine sont nombreux et variés : rétribution, expiation, intimidation (générale, spéciale), réparation du dommage, conciliation, neutralisation et réhabilitation;

- comme le droit pénal, la peine joue aussi une importante fonction morale et éducative;

- chaque sanction devrait viser un objectif principal, défini avec précision, qui serait fonction à la fois de sa nature spécifique (privative de liberté, pécuniaire, etc.), de la gravité de la conduite et de la personnalité de l'infracteur; elle pourrait viser également d'autres objectifs secondaires dans la mesure où ceux-ci ne sont pas incompatibles entre eux ou avec l'objectif principal;

- la peine doit être nécessaire et opportune; ceci veut dire qu'en l'imposant, le juge doit choisir la mesure la moins restrictive pour les libertés individuelles, faire preuve de modération (en particulier dans l'imposition d'une peine d'emprisonnement), utiliser davantage les mesures non carcérales (surtout celles axées sur la 
collectivité) et accorder une plus grande attention aux préoccupations des victimes et de la communauté;

- la peine doit être juste, équitable et proportionnelle à la gravité de l'infraction, au préjudice subi ou à subir et au degré de culpabilité du contrevenant;

- elle doit être efficace, c'est-à-dire atteindre les objectifs pour lesquels elle a été prévue;

- la structure actuelle des peines doit être améliorée grâce à l'acceptation de nouvelles mesures pénales, au regroupement, à la stratification hiérarchique et à la rationalisation des dispositions existantes et à la révision des maximums et des minimums prévus pour chaque infraction;

- la peine devrait entraîner les coûts économiques, financiers, sociaux et moraux les plus bas possibles;

3. La procédure pénale

- le principal objectif de la procédure pénale est l'harmonisation de deux autres objectifs : la défense de la société et la protection des libertés individuelles;

- le procès pénal doit obéir aux principes de légalité, égalité, équité, présomption d'innocence, doute raisonnable et respect des droits de la défense (protection contre l'arrestation et la détention arbitraires ainsi que contre les peines cruelles et inusitées, droit à une audience publique devant un arbitre indépendant et impartial, etc.).

4. Le système de justice pénale

- les objectifs du système de justice pénale doivent refléter ceux de ses composantes et tenir compte du caractère total du système;

- il doit y avoir des mécanismes appropriés de surveillance de son fonctionnement et de participation de la collectivité.

\section{B. SON APPLICATION DANS LE CAS DES DROGUES}

Une fois déterminé le modèle à suivre, il faudrait réviser les diverses normes et pratiques pénales en vigueur afin d'évaluer leur adaptation at modèle et de procéder à leur éventuelle correction. Dans ce but, la méthode la plus adéquate semble être l'analyse socio-historique de l'origine, de l'évolution et de la signification des législations et des pratiques pénales antérieurement indiquées. 
Examinons quelques résultats en matière de drogues susceptibles d'éclairer la portée de certains éléments du modèle.

\section{Justification}

En ce qui concerne tout d'abord la justification des législations pénales dans ce domaine précis, nous avons déjà mentionné qu'elles doivent refléter des nécessités sociales réelles et importantes.

Or, à la question «la drogue constitue-t-elle un problème social réel et important?», on peut répondre, de façon schématique, ce qui suit :

- De nombreux peuples ont connu et consommé des stupéfiants depuis des temps immémoriaux et dans des buts divers, sans cependant susciter des inquiétudes particulières;

- Jusqu'au XVIII' siècle, l'intervention de l'État se fait essentiellement pour des raisons religieuses et, à un degré moindre quoique significatif, pour des considérations de nature sanitaire et économique;

- Malgré un usage relativement large parmi certains secteurs de la population de plusieurs pays européens (France, Angleterre), il n'y a pas d'inquiétude sociale à l'égard des groupes jusqu'à la moitié du XIX ${ }^{e}$ siècle;

- Au XIX ${ }^{e}$ siècle, l'intervention de l'État semble surtout être due à des raisons économiques et politiques (défense de la liberté du commerce, colonisation, enrichissement rapide et considérable des personnes et des gouvernements aux dépens des colonies), le recours à la guerre afin d'atteindre ces objectifs n'étant pas par ailleurs écarté (l'exemple le plus connu est celui des célèbres guerres de l'opium). Vers la fin du siècle, cette intervention se justifie dans divers pays (Grande-Bretagne, ÉtatsUnis) pour des motifs d'ordre moral;

- Avec l'apparition des premières tentatives étiologiques de la dépendance et des premières expériences thérapeutiques, l'argument de la protection de la santé (individuelle et collective) pour justifier le jus puniendi dans ce domaine se manifeste de nouveau. Aux États-Unis, cet argument, invoqué par les premières associations de médecins et des pharmaciens, constitue la base de l'origine du "problème social» de la drogue et de la formation d'un consensus on a utilisé dès le début l'argument consistant à relier 
l'usage de la drogue à certains secteurs de la population (les Chinois, les Noirs et plus tard les Mexicains et les Portoricains aux États-Unis; les Chinois au Canada) ainsi qu'à la criminalité;

- $\mathrm{Au} \mathrm{XX}$ siècle, la lutte contre la drogue représente pour les États-Unis un excellent prétexte pour apparaître et s'affirmer sur la scène internationale et pour exercer une influence considérable aussi bien sur les premières législations sur les drogues promulguées à ce dernier niveau que sur les législations nationales des pays (dans la mesure où celles-ci s'inspirent de celles-là);

- Dès 1962, une approche non pénale en matière de drogues commence à se manifester dans ce pays, le drogué étant considéré non pas comme un délinquant mais comme un malade qui a besoin d'aide et de traitement;

- Une véritable préoccupation sociale pour le problème de drogue apparaît aux États-Unis vers les années 60, surtout comme une conséquence de l'étendue des mouvements de protestations des jeunes contre divers événements sociaux et politiques survenus dans ce pays (guerre du Vietnam, discrimination raciale, assassinat des frères Kennedy et du pasteur Martin Luther King, utilisation massive de marihuana et de LSD, etc.). La préoccupation vis-à-vis de ce phénomène est d'autant plus grande que ses principaux protagonistes sont les jeunes des classes moyenne et élevée, dont les conduites ne correspondaient plus aux valeurs traditionnelles américaines. La même situation se manifeste en France à l'occasion de la «révolution» de mai 1968, ainsi que dans d'autres pays européens;

- Au cours des 20 dernières années, les pénalités dans ce secteur ont augmenté considérablement, mais diverses tentatives visant la décriminalisation de la simple possession de marihuana ont également lieu;

- Ainsi, en ce qui concerne les États-Unis et certains pays européens et avec les nuances que nous indiquerons plus loin, nous pouvons constater l'existence d'un problème social par rapport à l'usage non médical des drogues. Dans d'autres pays, par contre, la préoccupation vis-à-vis de ce phénomène, ou bien n'a pas de justification empirique, ou bien a souvent été «importée» voire «imposée» par l'influence ou la pression des États-Unis. En Amérique latine, par exemple, l'intérêt manifesté par certains gouvernements en matière de drogues peut être considéré dans de nombreux cas comme une manœuvre de diversion destinée à 
attirer l'attention de la population sur ce phénomène, la détournant d'autres problèmes sociaux plus graves et urgents. En Colombie, les publications officielles font régulièrement allusion à de "grandes quantités» de jeunes consommateurs, attribuant leur conduite au problème de la contestation des jeunes envers le système socio-politique, alors que les recherches sur les charactéristiques de l'usage des stupéfiants dans ce pays montrent l'aspect relatif de cet usage; au Venezuela et à Costa Rica, la préoccupation sociale en matière de drogues semble être due à la diffusion des publications sur ce thème effectuée par les États-Unis par l'intermédiaire de leur ambassade, qui servent de modèle pour l'élaboration de brochures et instruments semblables;

- Sans prétendre ignorer l'existence d'un problème social relatif aux drogues dans certains pays, il convient toutefois d'indiquer ce qui suit : a) il est assez difficile d'évaluer avec précision le nombre de pharmaco-dépendants existant dans chaque pays, les caractéristiques de la consommation des stupéfiants, les conséquences de cet usage et leurs coûts (individuels, sociaux); b) prétendre isoler le problème de la drogue sans le placer sur le même plan que d'autres thèmes sociaux dont l'étendue, le danger et les coûts sont en général supérieurs (alcool, tabac, abus de médicaments, accidents de la route et du travail, etc.) constitue à la fois une erreur, une hypocrisie et une injustice; c) on ne peut pas accepter sans critique l'affirmation selon laquelle la drogue constitue un problème social, puisque dans des nombreux sondages d'opinion il ne figure pas parmi les sujets les plus préoccupants pour le public;

- On affirme généralement qu'il existe une corrélation statistique positive entre l'usage des drogues douces et des drogues fortes. Cette hypothèse de l'«escalade» n'a pas pu être vérifiée de façon scientifique dans la mesure où elle suppose l'existence du phénomène de dépendance physique ou psychique par rapport aux drogues douces, telle la marihuana, alors que ce phénomène ne semble pas se manifester dans le cas de ce type de substances. Par contre, ce qui semble avoir été démontré c'est que très souvent la marihuana constitue la première drogue illicite prise par les polytoxicomanes. Cependant, nous ignorons encore le rôle joué par le cannabis dans le passage à d'autres drogues; nous ne connaissons pas non plus aucune caractéristique de ce produit qui puisse engendrer la nécessité ou le désir de consommer d'autres substances. Ce sont les facteurs reliés au dynamisme personnel et 
aux transformations sociales qui semblent jouer un rôle prépondérant dans les modalités d'usage des drogues multiples, alors que l'action elle-même de ces produits n'intervient qu'à un degré moindre;

- Finalement, il existe une forte controverse sur les rapports entre l'usage des drogues et la criminalité; bien que l'on puisse constater d'importantes analogies entre ces deux phénomènes, l'existence d'une relation causale entre l'usage des drogues et la criminalité n'a pas encore été établie.

Par conséquent, les observations précédentes nous empêchent de donner une réponse totalement positive et générale à la question «la drogue constitue-t-elle un problème social réel et important?».

Examinons maintenant une autre question importante : celle $\mathrm{du}$ bien juridique que les législations pénales sur les drogues essaient de protéger en prohibant certaines conduites de nature à causer un grave dommage aux personnes ou à la collectivité.

\section{Objectifs}

Pour de nombreux auteurs, la principale justification de l'intervention pénale de l'État dans le secteur des drogues part de la prémisse selon laquelle la vie et la santé (de l'usager) constituent des biens ou des valeurs de première importance, la sanction pénale étant par conséquent nécessaire pour leur protection. Cette orientation fait de l'usage des stupéfiants le centre de la possible régulation de l'État, le reste des conduites du «cycle de la drogue» (tels les actes de culture, transport, vente ou trafic) devant être considérées comme de simples formes de participation à l'acte principal; elle polarise en outre l'analyse dans une double direction: proposer l'incrimination des substances susceptibles de mettre en danger la vie ou la santé, et recommander que les produits inadéquats pour cette fin demeurent en dehors des limites de l'intervention pénale.

Par rapport à ce sujet, on reconnaît le caractère nuisible pour la santé des substances opiacées, des amphétamines et des hallucinogènes. La discussion se limite donc à la cocaïne et au cannabis, dont le caractère dommageable pour la santé a tellement été mis en doute que, pendant les années 70 , de nombreux spécialistes avaient préconisé la légalisation de leur usage. Cependant, des recherches plus récentes montrent l'existence d'importants changements physiologiques et psychiques provoqués par l'usage prolongé de ces deux produits. 
Contre les arguments relatifs au caractère nocif des drogues, on peut toutefois invoquer les considérations suivantes: a) la plupart de ces substances produisent également des effets positifs (applications médicales et thérapeutiques, augmentation du plaisir et de la capacité pour la méditation, contribution à l'amélioration des relations sociales, etc.); b) leur usage modéré, surtout dans les cas des drogues douces, est tolérable chez les personnes saines et jeunes; c) l'absence de toute réaction pénale vis-à-vis d'autres produits beaucoup plus nuisibles et socialement répandus (tabac, alcool, etc.); d) les sociétés contemporaines reconnaissent qu'un individu normal est libre de décider sur sa propre vie et sa propre santé (en ce sens, la tentative de suicide n'est pas en général considérée comme un délit) ; par conséquent, il n'y a pas de raison d'adopter une attitude contraire lorsqu'il s'agit d'un usager de substances toxiques.

À leur tour, ces arguments ont été critiqués par plusieurs auteurs. D'un côté, on affirme que la persistance de l'incongruité relative à l'acceptation culturelle de l'alcool, du café et du tabac ne constitue pas un argument déterminant pouvant servir à fonder la thèse de la permissivité ou de la passivité vis-à-vis du phénomène de la drogue. Quant à l'argument de la liberté dont jouissent les individus de décider de leur propre vie et de leur propre santé, on lui oppose la référence à la santé physique et mentale de la population, véritable objet de la protection pénale; ceci élimine la question du consentement du consommateur, qui n'est plus titulaire de ce dernier bien juridique, éminemment social.

De tout ce qui vient d'être exposé nous pouvons déduire que, si le droit pénal doit toujours représenter l'ultima ratio du législateur, la nécessité d'une intervention d'une telle nature ne semble pas pleinement justifiée en matière de drogues, surtout si l'on tient compte des conséquences que l'intervention pénale de l'État a apportées jusqu’à présent dans ce champ.

\section{Portée et limites}

Ces conséquences se manifestent surtout sur la plan de l'application concrète - souvent sur celui de l'impossibilité d'une telle application - des dispositions pénales en matière de drogues.

En effet, dans de nombreux cas, la loi pénale est appliquée de façon incohérente, injuste et discriminatoire. Au Canada, par exemple, 90 à $95 \%$ des accusations portées pour violation de la Loi sur les stupéfiants se réfèrent régulièrement au cannabis, dont 
$85 \%$ à la simple possession de cette substance; les pourcentages sont semblables en ce qui concerne les déclarations de culpabilité. Au début des années 70 , la Commission d'enquête sur l'usage des drogues à des fins non médicales considérait que la pratique répressive dans ce domaine était discriminatoire, puisqu'elle n'atteignait que $0,5 \%$ des usagers connus. Dix années après, la situation ne semble pas avoir changé, et l'on peut même observer que : a) les peines applicables dans ce secteur figurent parmi les plus sévères prévues dans la législation pénale, n'étant surpassées que par celles qui punissent l'homicide; b) il y a entre elles des disparités injustifiables (par exemple, la peine maximale prévue pour le cas d'importation de n'importe quelle quantité de LSD est de 10 ans de pénitencier, alors que l'importation de marihuana - même d'une petite quantité - peut entraîner l'emprisonnement à vie, avec un minimum obligatoire de 7 ans de privation de liberté en cas de déclaration de culpabilité, ce qui constitue une situation irrationnelle dans la mesure où le premier produit est plus nocif que le second) ; c) toute personne accusée de trafic de stupéfiants jouit des privilèges traditionnels accordés à n'importe quel inculpé (dont celui qui consiste à imposer à l'accusation le fardeau de la preuve) ; par contre, lorsqu'il s'agit de simple possession, le législateur a inversé ce principe fondamental, de telle sorte qu'il appartient au possesseur de drogues de prouver devant le tribunal que la possession n'a pas pour objet le trafic.

Dans d'autres cas, les tentatives d'application de la loi pénale en matière de drogues ont entrainé - et continuent à entraîner d'importants effets "pervers» en ce qui concerne le fonctionnement du système pénal : élargissement des pouvoirs policiers, création de services spécialisés, mobilisation de policiers et de magistrats au détriment d'autres fonctions, recours à des procédures pénales inhabituelles pour faire respecter la loi (indicateurs de police, agents provocateurs), corruption de fonctionnaires policiers et judiciaires, augmentation de la population pénitentiaire et apparition du problème des drogues dans les prisons, hausse du nombre de délits reliés à l'usage illégal des stupéfiants, etc.

Par ailleurs, certains facteurs rendent difficile, voire inefficace l'application des normes pénales dans ce domaine. Ces facteurs concernent parfois le système de justice pénale (nombre relativement rare de dénonciations, détentions et condamnations; conflits entre les divers services policiers qui s'occupent de ce problème; effet dissuasif limité des sanctions pénales). D'autres se réfèrent 
aux relations déficientes qui existent entre les services judiciaires et sanitaires (parmi d'autres raisons, elles sont dues à une méfiance réciproque, au secret professionnel, au manque de services, au caractère inadéquat de ceux-ci). Finalement, les facteurs d'ordre économique et politique sont ici d'une importance capitale. En Colombie, par exemple, on estime à un milliard de dollars c'est-àdire, $40 \%$ du budget national) les revenus annuels provenant des exportations clandestines de marihuana, dont vivent quelques 40000 familles et environ 600000 personnes. Sans prendre en considération les sommes astronomiques que le gouvernement pourrait toucher et consacrer à combattre d'autres problèmes sociaux (chômage, logement, écoles, etc.) si la culture, le commerce et la possession de cannabis étaient légalisés, l'intervention pénale de l'État colombien, en plus d'avoir occasionné d'importants effets de nature économique (consolidation du pouvoir de l'économie souterraine, flux migratoire intense, inflation) ou politique (création d'un État de facto sous le principe de la guerre et de la fraude), a contribué à faire croître la contrebande d'armes chez les individus qui défendent la production et le trafic de marihuana et provoqué des vagues de violence entre les diverses bandes de trafiquants. Devant ces faits, les interventions des pouvoirs publics sont inopérantes, comme le montre le récent assassinat du ministre de la Justice, responsable d'une campagne vigoureuse contre le réseau de trafiquants de cocaïne installés au pays.

La situation colombienne ne constitue que l'illustration de celle qui existe dans presque tous les pays producteurs de drogues - la plupart d'entre eux appartiennent au Tiers Monde - et pose le problème beaucoup plus large et complexe du développement socio-économique dans le monde et de la domination que certains États exercent sur d'autres.

Par conséquent, les normes pénales sur les drogues, non seulement n'ont pas prouvé leur efficacité (le nombre de consommateurs n'a pas diminué, la production et le trafic ont augmenté), mais encore elles ont entrainé d'importantes conséquences négatives en prétendant résoudre ce problème.

\section{CONCLUSION}

Au terme de ces réflexions sur le droit de punir, l'impression dominante qui reste est celle d'un long chemin à parcourir. Sa première étape devrait être celle de la rigueur conceptuelle et 
théorique. Nous avons commencé cet essai en soulignant un certain nombre de confusions voire d'incohérences sur ce plan et en proposant un modèle ayant pour objectif de les éliminer - ou du moins de les réduire considérablement - . Ce modèle reflète en principe les tendances les plus récentes et réalistes en matière pénale des pays occidentaux les plus progressistes. Il s'agit, par conséquent, d'un modèle dont la plupart des éléments ont été «testés». Certains aspects restent néanmoins objet de controverses; tel est le cas des objectifs de la peine. Il pourrait donc, toujours en principe, être accepté, suivi et appliqué dans notre pays. Il l'est d'ailleurs, du moins dans ses grandes lignes directrices, malgré les confusions et les ambiguittés auxquelles nous avons déjà fait allusion. Voici donc une deuxième étape importante : celle impliquant une ferme décision politique à cet égard. Finalement, une troisième phase s'avère fondamentale. Elle aurait comme but principal la révision des normes et des pratiques pénales en vigueur afin de les adapter au modèle proposé et de leur donner une nouvelle rationalité.

Cette dernière étape est particulièrement ardue. Quelques questions, à titre d'exemple, témoignent de cette difficulté. Si le modèle s'appliquait à tous les délits d'une certaine gravité - ils pourraient être fort nombreux - comment déterminer le quantum des éléments qui devraient être réunis pour obtenir à la fois une définition socialement acceptable des conduites à prohiber pénalement et une intervention adéquate? Ces éléments devraient-ils être nécessairement les mêmes pour chaque type d'infraction? Quel devrait être le poids relatif de chacun d'eux? Comment établir de façon concrète l'équilibre entre certains objectifs contradictoires (protection sociale $v s$ droits de la défense, justice $\nu s$ efficacité)?

L'existence de ces difficultés ne devrait pas, à notre avis, constituer un prétexte pour l'inaction. L'esprit humain a montré, dans d'autres secteurs et tout au long de l'histoire, sa vigueur et sa capacité dans la solution des problèmes complexes. Au risque de se tromper, mais aussi en progressant constamment grâce à ses erreurs. Tel est le prix à payer si nous voulons avoir un jour un système pénal capable de répondre de façon satisfaisante à l'aspiration sans cesse renouvelée de justice qui a toujours caractérisé l'espèce humaine. 